Journal of Animal and Veterinary Advances 11 (16): 2923-2925, 2012

ISSN: $1680-5593$

(C) Medwell Journals, 2012

\title{
AFM and LSCM Studies of Choriocarcinoma Cell Line JAR Infected by Hepatitis B Virus
}

\author{
${ }^{1}$ Lu Gan, ${ }^{2}$ Ming Wei, ${ }^{1}$ Caiping Ke, ${ }^{1}$ Zhao Zhang and ${ }^{1}$ Xiaomin Xiao \\ ${ }^{1}$ Department of Obstetrics and Gynecology, First Affiliated Hospital of Jinan University, \\ 510632 Guangzhou, China \\ ${ }^{2}$ Department of Basic Medical, Xian Medical University, 710071 Xian, China
}

\begin{abstract}
Choriocarcinoma cell line JAR was used as a model to study trophoblast. In this study, the morphological changes and three-dimensional reconstruction of JAR infected by Hepatitis B Virus (HBV) were detected with the use of Atomic Force Microscope (AFM) and Laser Scanning Confocal Microscope (LSCM). It was clearly shown that the HBsAg located in the cytoplasm or at the edge of the nucleus of JAR cells infected by HBV, the membrane of JAR cells infected by HBV were depressed, boundaries of cytoplasm and nucleus were unclear. The membrane of JAR were covered by particles and the histogram of particles in the negative group distributed from $400-800 \mathrm{~nm}$ in diameter, most of paticles were $800 \mathrm{~nm}$ while the particles of JAR cells infected by HBV ranged from $200-600 \mathrm{~nm}$, most of paticles were $400 \mathrm{~nm}$. HBV serum can infect JAR cells in vitro, the changes of the morphology of JAR cells infected by HBV were related to the damage of the particles on the membrane of JAR cells. AFM can be useful for studying cell pathology.
\end{abstract}

Key words: Choriocarcinoma cell line JAR, hepatitis B virus, atomic force microscope, laser scanning confocal microscope, cell pathology

\section{INTRODUCTION}

China is a high prevalence area of hepatitis $B$, hepatitis B surface antigen carrier rate is as high as $10-15 \%$ (Ocama and Opio, 2005). It's well known that perinatal transmission of $\mathrm{HBV}$ is a major cause of chronic hepatitis, cirrhosis and hepatocelluar carcinoma (Chang, 2007). So far, the mechanism of HBV vertical transmission is still unclear. Some studies indicated that HBV can infect not only liver-derived cells but also non-hepatic cells. Atomic force microscope is a kind of new experimental technique to observe the morphology of cells with the interaction between atomic and molecular (Domke et al., 2000). The cells can be three-dimensional reconstructed by AFM. There is still no report about the changes of surface structure of cells infected by HBV in vitro. In this study, researchers use HBV serum to infect choriocarcinoma cell line JAR, to observe the morphological changes and 3D reconstruction of JAR cells infected by HBV with the use of atomic force microscope and laser scanning confocal microscope.

\section{MATERIALS AND METHODS}

Regents and chemicals (Cells and reagents): Choriocarcinoma cell line JAR as a trophoblast model was purchased from cell resource center in Shanghai.
HBV-DNA serum: collect the serum of HBV carriers whose HBV-DNA copy was $5 \times 10^{8}$ and the serum of healthy people whose HBV serologic marker was negative. The sera were filtration-sterilized with $0.22 \mu \mathrm{m}$ filters. RPMI-1640 medium with high glucose, GIBCO, 2.5\% glutaraldehyde fixative, Guangzhou Chemical Reagent Factory.

JAR cells culture: JAR cells were grown in RPM 1640 supplemented with $10 \%$ fetal bovine serum and antibiotics (penicillin $100 \mathrm{U} \mathrm{mL}^{-1}$ and streptomycin $100 \mathrm{mg} \mathrm{mL}^{-1}$ ). The cells were seeded into a concentration of $2 \times 10^{5}$ cells $\mathrm{mL}^{-1}$ in flasks. The medium was changed every 2 days. The cultured cells were observed daily with a phase-contrast microscope. When JAR cells reached a $30-50 \%$ confluence, treated with the virus serum at $37^{\circ} \mathrm{C}$ under a $5 \% \mathrm{CO}_{2}$ atmosphere for $24 \mathrm{~h}$.

Immunofluorescent staining: JAR cells were washed trice with PBS and then fixed in ice-cold 95\% ethanol for $20 \mathrm{~min}$ at room temperature. The slides were air-dried for $20 \mathrm{~min}$ then stored at $80^{\circ} \mathrm{C}$ until assayed or immediately stained for HBsAg. For the staining, the cells were washed 3 times with PBS. Endogenous peroxidase activity was neutralized with a $30 \mathrm{~min}$ incubation at room temperature with $3 \%$ Triton-X100, followed by a $1 \mathrm{~h}$ incubation at room temperature in $10 \%$ nonimmune goat serum to block

Corresponding Author: Xiaomin Xiao, Department of Obstetrics and Gynecology, First Affiliated Hospital of Jinan University, 510632 Guangzhou, China 
nonspecific sites. A primary antibody (Zhong Shan Jin Qiao Company, Beijing) was added to the solution which is able to bind to HBsAg (diluted 1:50); the slides were incubated overnight at $4^{\circ} \mathrm{C}$. Then, the slides washed with PBS and incubated with FITC-conjugated secondary antibody (Zhong Shan Jin Qiao Company, Beijing) for $30 \mathrm{~min}$. The JAR cells were observed by laser scanning confocal microscope (Zeiss LSM 510 Germany).

AFM imaging: PBS wash for $5 \mathrm{~min} \times 2$ times; $2.5 \%$ glutaraldehyde $20 \mathrm{~min}$; triple-distilled water wash for 10 min $\times 3$ times; natural drying in air. The samples were fixed on the the xy scanning stage of AFM (CP-Research, USA). Using $100 \mu \mathrm{m}$ scanner, contact mode, UL20B silicon probe. Software (IP2.1) for acquisition the images and data and all images were processed smooth.

\section{RESULTS AND DISCUSSION}

HBsAg in JAR cells infected by HBV: HBsAg was founded in JAR cells infected by HBV serum. The HBsAg located in the cytoplasm and at the edge of the nucleus. HBsAg was not founded in JAR cells in the negative control group (Fig. 1a and b).

Three-dimensional reconstruction of JAR cells infected by HBV: Cells contacted with the surrounding environment through the extension of pseudopodia, to draw nutrients and pass signals between cells to form community effects. Infected by $\mathrm{HBV}$, the membrane of JAR cells were depressed, boundaries of cytoplasm and nucleus were unclear, pseudopodia disappeared. JAR cells in the negative control group were elongated spindle or polygonal, the boundaries of cytoplasm and the nucleus were clear and the volume of the nucleus was large, full and convex. There were a lot of pseudopodia around the membrane. These indicated that JAR cells were in the active state of growth (Fig. 2).

The change of ultrastructure of choriocarcinoma JAR cells infected by HBV: Particles of JAR cells in the negative group distributed from $400-800 \mathrm{~nm}$ in diameter, most of paticles were $800 \mathrm{~nm}$ while the particles of JAR cells infected by HBV ranged from 200-600 nm, most of paticles were $400 \mathrm{~nm}$. The particles of JAR cells infected by HBV became smaller than the negative group. The particles on the membrane of cells may be proteins, protein-lipid complexes and the protrusions and microvilli (Le Grimellec et al., 1998). Changes in the external environment will lead to actin protein, adhesion protein on membrane of cells reconstruct and rearrange in order to communicate with the extracellular matrix

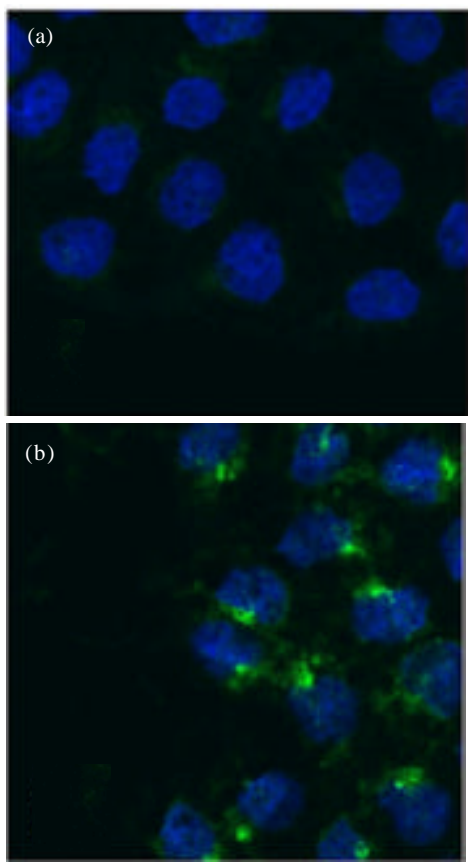

Fig. 1: LSCM image of JAR cells infected by HBV. a) The JAR cells in the negative group and b) the JAR cells infected by HBV

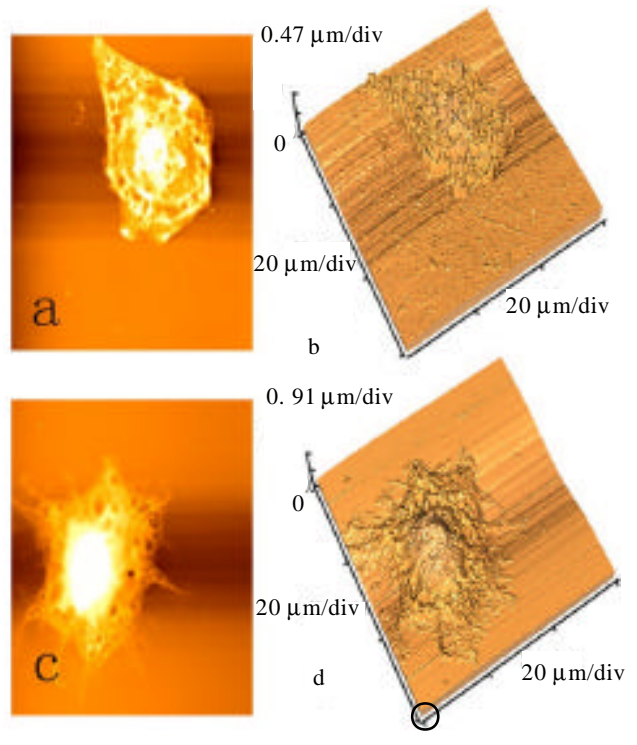

Fig. 2: AFM image of three-dimensional reconstruction of JAR cells infected by HBV; $a$ and b) the JAR cells infected by HBV, c and d) the JAR cells in the negative group

(Radmacher, 2002). The results suggested that HBV infection of JAR cells may be related to destruction of these structures on the membrane of cells (Fig. 3a-d). 


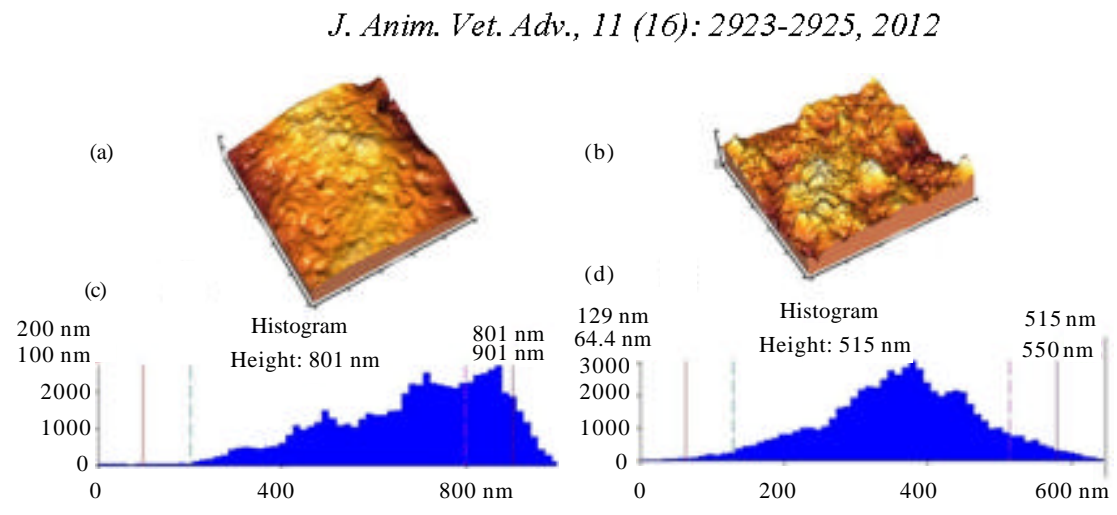

Fig. 3: AFM image of ultrastructure of JAR cells infected by HBV; $a$ and b) three-dimensional ultrastructure $(5 \times 5 \mu \mathrm{m})$ of the membrane on JAR cells. $\mathrm{c}$ and d) Histogram showing the particles size distribution of membrane. c) Particles of JAR cells distribute from $400-800 \mathrm{~nm}$ in diameter, most of particles is $800 \mathrm{~nm}$. d) The diameter of JAR cells infected by HBV particles range from $200-600 \mathrm{~nm}$, most of paticles is $400 \mathrm{~nm}$

\section{CONCLUSION}

AFM is used as a powerful tool for detecting ultrastructures of cells. In this study, the membrane of JAR cells infected by HBV were depressed, boundaries of cytoplasm and nucleus were unclear. The membrane of JAR cells were covered by particles and the histogram of particles size in the negative group distributed from $400-800 \mathrm{~nm}$ in diameter, most of paticles were $800 \mathrm{~nm}$ while the particles of JAR cells infected by HBV ranged from 400-800 nm, most of paticles were $400 \mathrm{~nm}$ (Fig. 3). The particles of JAR cells became smaller because of HBV infection. By LSCM, it was clearly shown that the HBsAg located in the cytoplasm or at the edge of the nucleus of JAR cells infected by HBV. Combining the result of images of AFM and images of FSCM, researchers can note that $\mathrm{HBV}$ can infect JAR cells in vitro and the changes of the morphology of JAR cell infected by HBV were related to the damage of the particles on the membrane of the cells. AFM can be useful for studying cell pathology.

For the first time that we know of, researchers have observed the morphological changes and 3D reconstruction of JAR cells infected by HBV serum by atomic force microscope, specifically the changes of ultrastructure on the membrane of JAR cells.

\section{ACKNOWLEDGEMENTS}

This research was supported by grant from the Ministry of Education of China (20070559006) and the National Natural Science Foundation of China (C30973214).

\section{REFERENCES}

Chang, M.H., 2007. Hepatitis B virus infection. Semin. Fetal. Neonatal. Med., 12: 160-167.

Domke, J., S. Dannohl, W.J. Parak, O. Muller, W.K. Aicher and M. Radmacher, 2000. Substrate dependent differences in morphology and elasticity of living osteoblasts investigated by atomic foroe microscopy. Colloids. Surf. B. Biointerf., 19: 367-379.

Le Grimellec. C., E. Lesniewska, M.C. Giocondi, E. Finot, V. Vie and J.P. Goudonnet, 1998. Imaging of the surface of living cells by low-force contact-mode atomic force microscopy. Biophys. J., 75: 695-703.

Ocama, P. and C.K. Opio, 2005. Lee WM. Hepatitis B virus infection: Current status. Am. J. Med., 118: 1413-1413.

Radmacher, M., 2002. Measuring the elastic properties of living cells by the atomic force microscope. Methods. Cell. Biol., 68: 67-90. 